\title{
LA CRECIENTE RELEVANCIA DEL BINOMIO SEGURIDAD/INSEGURIDAD COMO PREOCUPACIÓN DE ORDEN PÚBLICO: ¿UN FENÓMENO ARGENTINO?
}

\author{
Germán Oliveto ${ }^{1}$
}

En los últimos años el binomio seguridad/inseguridad se ha consolidado como una de las preocupaciones de orden público más relevante para la mayoría de los argentinos. Este tema se ha instalado en la agenda de los medios de comunicación masiva así como en el centro del debate político de nuestro país. La determinación de las causas, los responsables y las soluciones, así como la real envergadura del problema son motivo de controversia entre los académicos y especialistas en la materia.

El presente análisis dejará de lado el debate sobre la existencia (o no) de una tendencia incremental de los hechos delictivos en el país. Las razones de esta decisión son variadas: en primer lugar, existen serias dificultades para acceder a estadísticas confiables debido a lo que se conoce como la "cifra negra de la criminalidad" -esto es, la brecha entre los hechos delictivos ocurridos y los efectivamente denunciados y registrados-, a lo que se suma la cuestión del "carácter manufacturado" de las estadísticas oficiales, es decir, la complejidad de que sea la propia institución policial y judicial la encargada de su registración y oficialización. Como respuesta a estos problemas, han proliferado las llamadas "estadísticas de victimización", pero estas han sido discontinuadas en el tiempo y han variado en sus

\footnotetext{
${ }^{1}$ Germán Oliveto es licenciado en sociología, docente en la Facultad de Ciencias Sociales de la Universidad de Buenos Aires y maestrando en Investigación en Ciencias Sociales (UBA). Actualmente se desempeña como consultor independiente y director de proyectos para la consultora MBC MORI. Email: germanoliveto@yahoo.com.ar
} 
instrumentos de medición, lo que dificulta su comparabilidad y su efectividad para medir tendencias (Sozzo, 2003). La segunda razón es que, como señala Kessler, el "sentimiento de inseguridad" es un hecho social diferenciado del crimen, con dinámicas y consecuencias sociales específicas. En ese sentido, exhibe una autonomía relativa, puesto que "suele aumentar al incrementarse la victimización pero una vez instalada como problema social, ya no disminuye aunque las tasas de delito lo hagan" (Kessler, 2011).

En definitiva, partimos de asumir que, con independencia de lo que las estadísticas sobre hechos delictivos señalen, lo que es evidente es que el binomio seguridad/inseguridad ocupa un lugar cada vez más importante entre las preocupaciones de la opinión pública y este es en sí mismo un hecho relevante que merece nuestra atención.

Al tratarse de un fenómeno social complejo, resulta conveniente analizarlo a partir de diferentes dimensiones, de manera de acercarnos a una conceptualización lo más completa posible sobre él. En este trabajo en particular nos proponemos abordar exclusivamente el interrogante sobre si la emergencia del binomio seguridad/inseguridad como preocupación de orden público se encuentra circunscripta a nuestro país o si, por el contrario, puede enmarcarse como parte de un fenómeno latinoamericano.

Analizar resultados y tendencias de importantes estudios de opinión pública desarrollados en la región nos dará el marco de esta indagación, que constituirá un pilar relevante en la identificación de las causas de la emergencia del tema en nuestro país.

\section{El tema en la agenda pública nacional}

El punto de partida de nuestra investigación pasa por ubicar la presencia del binomio seguridad/inseguridad como asunto relevante para la opinión pública del país. Esto puede constatarse en prácticamente cualquier estudio serio de investigación social aplicada que indague sobre las preocupaciones de la población en los últimos años, mediante 
abordajes ya sea cualitativos o cuantitativos. Pero no es solo su relevancia actual, es decir, su vigencia presente, lo que resulta interesante, sino también el que se trate de un fenómeno relativamente nuevo en términos de preocupación socialmente formulada. Dicho de otro modo: la seguridad/ inseguridad no siempre fue algo relevante para la opinión pública, más bien por el contrario, se trata de un tema que se ha expandido aceleradamente en un corto tiempo.

Para tal afirmación nos apoyamos en datos brindados por el Latinobarómetro, un estudio cuantitativo de opinión pública realizado en 18 países de América Latina desde 1995 hasta la actualidad. ${ }^{2}$

\section{Gráfico 1: Principal problema del país: Argentina}

Pregunta: En su opinión, ¿Cuál considera Ud. que es el problema más importante en el país?

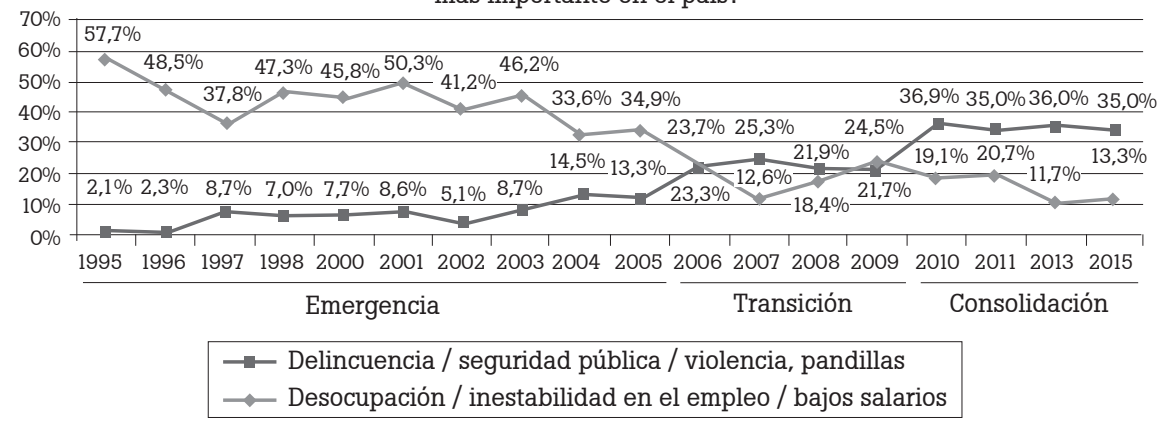

Fuente: Latinobarómetro

En el Gráfico $N^{0} 1$ podemos visualizar una tendencia en la que gradualmente los temas enmarcados en el binomio seguridad/inseguridad van desplazando a las preocupaciones vinculadas al empleo (desocupación/inestabilidad/salarios) dentro de las consideraciones sobre el "problema más importante del país". Cabe destacar que la pregunta aquí ana-

\footnotetext{
${ }^{2}$ En Argentina se realizan anualmente 1200 encuestas en todo el país con una muestra nacional probabilística, estratificada y con cuotas de sexo y edad para la selección final del entrevistado en el hogar.
} 
lizada fue de respuesta abierta y luego codificada en función de categorias establecidas por Latinobarómetro. Por su parte, el agrupamiento de temas aquí presentado es de elaboración propia, pero resulta pertinente aclarar que en ninguna medición hubo otra categoría (o conjunto de categorías lógicamente agrupables) con más menciones que las indicadas en el gráfico $\mathrm{N}^{\circ} 1$. Dicho en otros términos, las cuestiones vinculadas al empleo primero y a la seguridad/inseguridad después han recibido en los últimos veinte años más menciones que temas tales como salud, educación, vivienda, corrupción, pobreza, medio ambiente e inflación, entre otros.

En el mismo gráfico observamos también que el desplazamiento en las preocupaciones de la opinión pública argentina ha resultado de un proceso gradual, pudiéndose distinguir analíticamente entre un "período de emergencia", donde nuestro tema de estudio pasa progresivamente de ocupar un lugar marginal en las primeras mediciones a cosechar cada vez más menciones hacia el 2004/2005. Un segundo momento, que podemos denominar como "transición", en el que tanto las menciones vinculadas a la seguridad/inseguridad como las agrupadas en las cuestiones del empleo comparten el podio de las preocupaciones más relevantes (2006/2009). Y, finalmente, un tercer momento, de "consolidación" del tema, donde este resulta claramente señalado como el más relevante por la mayor parte de los entrevistados (2010 en adelante).

Si bien los datos que analizaremos en este trabajo corresponden a un solo estudio, cabe señalar que la tendencia que ubica a la seguridad/inseguridad en el centro de las preocupaciones de la opinión pública en la actualidad puede verificarse en una gran cantidad de investigaciones contemporáneas. A modo de ejemplo podemos señalar que en el estudio LAPOP (Latin American Public Opinion Project), realizado por la Universidad de Vanderbilt, la "falta de seguridad" fue señalada como el problema más importante del país con el 22\% de las menciones, pero incluso otro 10\% indicó a la "delincuencia, crimen" situándose den- 
tro del mismo conjunto temático. ${ }^{3}$ También en febrero de 2014 la inseguridad se ubicaba en primer orden de preocupaciones según una medición de la consultora Raúl Aragón \& Asociados; ${ }^{4}$ asimismo, en abril de 2013 fue señalado como "el tema que más afecta al país" por el 23\% de los consultados en la encuesta de Expectativas Económicas (IGEE), que elaboran la Universidad Católica Argentina (UCA) y TNS Gallup Argentina. ${ }^{5}$

No obstante, las ventajas que nos impulsan a tomar aquí los datos del Latinobarómetro como punto de referencia pasan por: a) tratarse de un estudio sistemático realizado en el país desde hace 20 años, ${ }^{6}$ lo que permite identificar evoluciones y tendencias a lo largo del tiempo; b) contar con una muestra extendida y representativa de los diferentes estratos de la población; ${ }^{7}$ c) haber sido parte de la ejecución de dicho estudio en el pais; ${ }^{8} \mathrm{y}$ d) tratarse de un estudio internacional, lo que permite establecer comparaciones con otros paises. Es precisamente este último punto el que resulta de particular relevancia de cara a la indagación que nos proponemos en este trabajo, a saber, determinar si el fenómeno de emergencia del binomio seguridad/inseguridad como tema relevante para la opinión pública se restringe a nuestro país o si, por el contrario, es parte de una tendencia latinoamericana.

\footnotetext{
${ }^{3}$ http://datasets.americasbarometer.org/database-login/index.html

${ }^{4} \mathrm{http} / / /$ www.elpais.com.uy/mundo/argentina-inseguridad-jorge-capitanichgobierno.html

${ }^{5}$ http://www.eleconomistaamerica.com.ar/sociedad-eAm-argentina/noticias/ 4868528/05/13/Inseguridad-e-inflacion-los-principales-problemas-de-losargentinos.html

${ }^{6}$ El estudio no fue realizado en los años 1999, 2012 y 2014.

${ }^{7}$ Muchos estudios de opinión pública que se presentan como nacionales incluyen en verdad solo a los grandes centros urbanos (localidades de más de 500.000 habitantes), excluyendo así a una porción significativa de la población del país.

${ }^{8}$ Como director de proyectos de MBC MORI, empresa responsable por la realización del Latinobarómetro en la Argentina desde sus inicios, he tenido una participación directa en la coordinación del estudio, obteniendo así un conocimiento de primera mano sobre los procedimientos implementados y la información obtenida.
} 


\section{El contexto latinoamericano}

Numerosos economistas, sociólogos y politólogos han postulado a América Latina como el "continente de la esperan$z a$ " frente a un nuevo ciclo de crisis económica internacional que viene azotando al mundo entero y golpeando con particular virulencia en algunos de los países desarrollados. Los motivos para otorgarle tal denominación son variados y exceden en su complejidad los marcos del presente trabajo, pero en general podemos señalar dos pilares fundamentales: las cuantiosas reservas de recursos naturales, con una importancia estratégica de cara al futuro de la humanidad, y los relevantes procesos de movilización social y transformaciones políticas que se han sucedido desde comienzos de siglo en distintos países del continente, buscando establecer niveles de ruptura (de distinta intensidad según el caso) con las políticas del orden hegemónico global. En ese marco, algunos autores distinguen con más precisión entre dos tipos de procesos que coexisten en la región: por un lado, el de los países con gobiernos "posneoliberales" y de corte "neodesarrollista", los que "sin pretender producir en lo sustancial una ruptura explícita con las políticas neoliberales, imponen cambios de acento y nuevos énfasis tanto en materia social como en políticas de producción." (Gambina, 2013). Por otra parte, se encuentran los llamados "procesos de transición", que se definen por su búsqueda de una salida al neoliberalismo y el subdesarrollo, así como por "establecer una nueva articulación regional, diferente a la hegemónica, que haga posible la construcción de una nueva matriz productiva, social y política que desarticule el proceso de dependencia y altere el modo de producción". (Elorza, 2015).

Esta descripción resulta pertinente puesto que remite al proceso de reordenamiento político, económico y social transcurrido en la región durante el mismo período en que, según hemos analizado, se produce la emergencia del binomio seguridad/inseguridad como tema de orden público relevante en nuestro país. 
Volveremos más adelante sobre la mencionada distinción entre los dos tipos de procesos que coexisten en la región vinculándolo a nuestro tema de investigación. Pero retomemos ahora el interrogante sobre si el mencionado desplazamiento de las preocupaciones de la opinión pública se encuentra restringido a nuestro país o si, por el contrario, debe ser enmarcado como parte de un proceso al menos regional.

En primer lugar analizaremos los datos agregados a nivel continental del mismo estudio que utilizamos para visualizar la emergencia, transición y consolidación del tema en nuestro país, como forma de "medir con la misma vara", evitando así posibles distorsiones o sesgos producto del instrumento de medición. ${ }^{9}$

\section{Gráfico 2: Principal problema del país: Datos agregados América Latina}

Pregunta: En su opinión, ¿Cuál considera Ud. que es el problema más importante en el país?

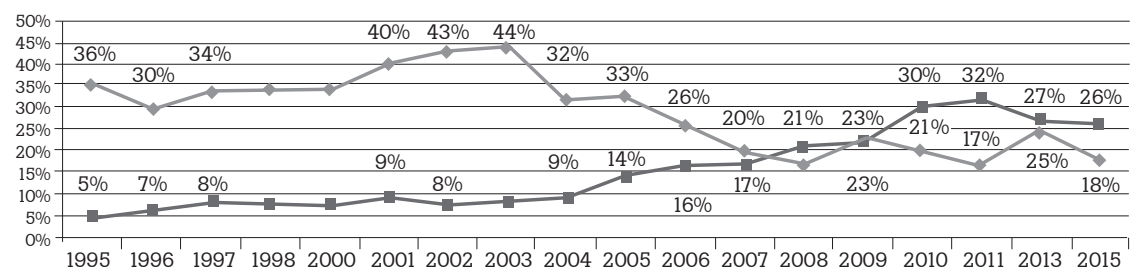

$\longrightarrow$ Desocupación / inestabilidad en el empleo / bajos salarios
$\longrightarrow$ Delincuencia / seguridad pública / violencia, pandillas

Fuente: Latinobarómetro

En el gráfico $N^{\circ} 2$ podemos ver la misma evolución histórica de los dos agrupamientos de temas analizados ante-

\footnotetext{
${ }^{9}$ El Latinobarómetro se aplica en 18 países de la región. Realiza anualmente alrededor de 20.000 entrevistas, representando de este modo a unos 600 millones de habitantes. Con algunas variaciones según el año, el estudio se lleva a cabo en: Argentina, Bolivia, Brasil, Chile, Colombia, Costa Rica, República Dominicana, Ecuador, El Salvador, Guatemala, Honduras, México, Nicaragua, Panamá, Paraguay, Perú, Uruguay y Venezuela.
} 
riormente para la Argentina. ${ }^{10}$ Las menciones vinculadas al empleo se muestran en la línea con rombos, mientras que los englobados en el tema seguridad/inseguridad están indicados con rectángulos. Podemos allí observar, en términos generales, la misma tendencia que habíamos mencionado para el caso argentino, donde el binomio seguridad/ inseguridad va progresivamente instalándose como tema relevante para la opinión pública latinoamericana.

Por su parte, con el gráfico $\mathrm{N}^{\circ} 3$ podemos comparar cómo se produce la evolución del binomio seguridad/inseguridad como tema relevante para la opinión pública en Argentina (línea con rombos) y en América Latina de forma agregada (línea con rectángulos). Allí podemos observar de forma aún más clara una tendencia similar, aunque debemos precisar que el salto del denominado período de emergencia al de transición es más pronunciado en el caso argentino (donde pasa del 13\% en 2005 al 23\% en 2006), mientras que, tomando los datos de América Latina de conjunto, este pasaje se produce en forma más gradual. El otro punto de distinción, en la misma línea del anterior, es que si bien el momento de consolidación del tema ocurre en el mismo período (2010 en adelante), en el caso de la Argentina es más pronunciado que en el de todo el continente, donde inclusive podemos visualizar en las últimas mediciones analizadas (2013-2015) una pérdida de peso del tema.

Así, podemos concluir que la emergencia y posterior consolidación del binomio seguridad/inseguridad como problema relevante para la opinión pública de nuestro país excede sus marcos y se sitúa como un fenómeno al menos latinoamericano.

\footnotetext{
${ }^{10}$ Siendo válidas las mismas salvedades realizadas al analizar el Grafico $\mathrm{N}^{\circ} 1$ en cuanto a la construcción de categorias por parte de Latinobarómetro y el posterior agrupamiento nuestro realizado ad-hoc para el presente análisis.
} 
Gráfico 3: Evolución del tema seguridad/inseguridad:

Comparativo Argentina-América Latina

Pregunta: En su opinión, ¿Cuál considera Ud. que es el problema más importante en el país?

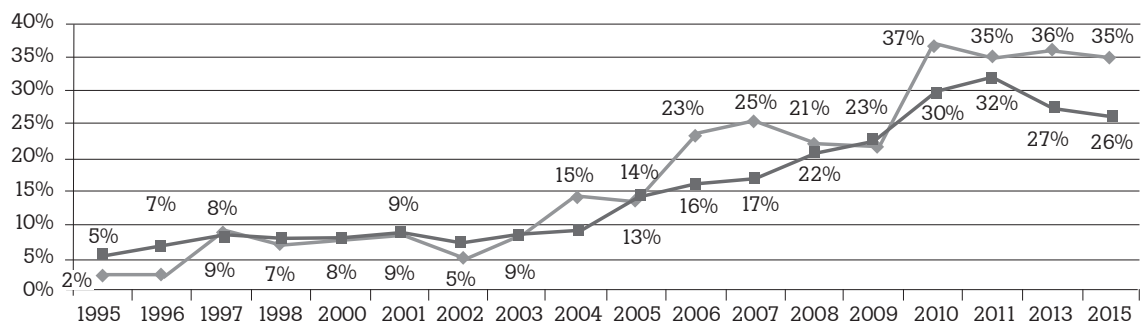

$\leadsto$ Argentina

$\rightarrow$ Total América Latina

Fuente: Latinobarómetro

\section{Similitudes y diferencias al interior de la región}

Hasta aquí hemos visualizado que el desplazamiento de las preocupaciones de la opinión pública encuentra un comportamiento similar a nivel continental al registrado en nuestro país. Pero es de esperar que la tendencia latinoamericana no sea homogénea en su interior y que muestre situaciones diferentes en cada país. Nos proponemos a continuación realizar un análisis comparado de los datos de los distintos países a fin de identificar si existen tendencias y comportamientos equivalentes.

Para ello tomaremos como punto de referencia los agrupamientos anteriormente mencionados a nivel continental, como forma de corroborar si existe correspondencia entre procesos que podemos identificar de similares características desde el punto de vista de su situación política, económica y social y las preocupaciones expresadas por sus respectivas poblaciones. Retomando a Gambina podemos entonces identificar por un lado un conjunto de países con gobiernos "posneoliberales" y de corte "neodesarrollista" (Gambina, 2013) entre los que se encuentran Brasil, Argentina y Uruguay, distinguiéndolos de los que Elorza denomina 
los "procesos de transición", portadores de "un discurso y un accionar anticapitalista y antiimperialista", entre los que el mencionado autor señala a Venezuela, Ecuador y Bolivia. ${ }^{11}$

Para establecer la presente comparación tomaremos en consideración solo a los mencionados países. No obstante, creemos pertinente realizar algunas aclaraciones y salvedades: a) En primer lugar, la comparación entre países resulta siempre dificultosa, producto de particularidades culturales y de idiosincrasia. Sin embargo, el hecho de que se trata del análisis de un mismo estudio otorga un marco de normas y procedimientos estandarizados que nos permite confiar en la comparabilidad de los resultados alcanzados; b) Cabe recordar que la dimensión que aquí estamos analizando (principal problema del país) es producto de una pregunta abierta posteriormente codificada por cada empresa consultora en función de categorias establecidas por Latinobarómetro. Ahora bien, para el presente análisis hemos condensado las categorías vinculadas a las cuestiones de empleo (desocupación /inestabilidad en el empleo/bajos salarios) así como las que podemos asociar al binomio seguridad/inseguridad (delincuencia/ seguridad pública / violencia, pandillas). Esto resulta relevante ya que, a diferencia de lo analizado en el caso de Argentina y América Latina, en algunos casos puntuales (en determinados años y países) otros temas habrian obtenido mayor cantidad de menciones de no ser por nuestra condensación posterior; c) a efectos de simplificar la exposición no mostraremos los porcentajes de cada tema, sino tan solo cuál ha sido en cada año el que obtuvo mayor cantidad de menciones al interior de cada país. De este modo, si bien perdemos en volumen de información, ganamos en claridad expositiva.

Realizadas las salvedades del caso, en el Cuadro 1 podemos observar la evolución temporal de las preocupacio-

\footnotetext{
${ }^{11}$ Elorza incluye dentro de este grupo a Cuba, aunque con las salvedades del caso por la particularidad de su proceso político, económico y social. No obstante, no incluimos al mencionado país en nuestro análisis ya que no disponemos de datos comparables sobre las preocupaciones de la opinión pública en él.
} 
nes al interior de cada país. Para facilitar su visualización sombreamos en gris las celdas cuando los temas vinculados al empleo obtuvieron la mayor cantidad de menciones, dejamos en blanco y con mayúsculas cuando lo hicieron las cuestiones englobadas en la seguridad/inseguridad y con minúscula y cursiva para otros temas.

Cuadro 1: Principal problema al interior de cada país y en América Latina de conjunto. Evolución temporal ${ }^{12}$

\begin{tabular}{|c|c|c|c|c|c|c|c|}
\hline Año & $\begin{array}{c}\text { América } \\
\text { Latina }\end{array}$ & Argentina & Brasil & Uruguay & Venezuela & Ecuador & Bolivia \\
\hline 1995 & EMPLEO & EMPLEO & EMPLEO & EMPLEO & inflación & S/D & S/D \\
\hline 1996 & EMPLEO & EMPLEO & EMPLEO & EMPLEO & educación & educación & educación \\
\hline 1997 & EMPLEO & EMPLEO & EMPLEO & EMPLEO & educación & EMPLEO & EMPLEO \\
\hline 1998 & EMPLEO & EMPLEO & EMPLEO & EMPLEO & educación & EMPLEO & educación \\
\hline 2000 & EMPLEO & EMPLEO & EMPLEO & EMPLEO & educación & EMPLEO & EMPLEO \\
\hline 2001 & EMPLEO & EMPLEO & EMPLEO & EMPLEO & EMPLEO & EMPLEO & EMPLEO \\
\hline 2002 & EMPLEO & EMPLEO & EMPLEO & EMPLEO & EMPLEO & EMPLEO & EMPLEO \\
\hline 2003 & EMPLEO & EMPLEO & EMPLEO & EMPLEO & EMPLEO & EMPLEO & EMPLEO \\
\hline 2004 & EMPLEO & EMPLEO & EMPLEO & EMPLEO & EMPLEO & EMPLEO & EMPLEO \\
\hline 2005 & EMPLEO & EMPLEO & EMPLEO & EMPLEO & EMPLEO & $\begin{array}{c}\text { situación / } \\
\text { problemas } \\
\text { de la politica }\end{array}$ & EMPLEO \\
\hline 2006 & EMPLEO & EMPLEO & EMPLEO & EMPLEO & SEGURIDAD & EMPLEO & EMPLEO \\
\hline 2007 & EMPLEO & SEGURIDAD & SEGURIDAD & EMPLEO & SEGURIDAD & EMPLEO & EMPLEO \\
\hline 2008 & SEGURIDAD & SEGURIDAD & SEGURIDAD & EMPLEO & SEGURIDAD & EMPLEO & $\begin{array}{c}\text { situación / } \\
\text { problemas } \\
\text { de la politica }\end{array}$ \\
\hline 2009 & EMPLEO & EMPLEO & EMPLEO & SEGURIDAD & SEGURIDAD & EMPLEO & EMPLEO \\
\hline 2010 & SEGURIDAD & SEGURIDAD & salud & SEGURIDAD & SEGURIDAD & EMPLEO & $\begin{array}{l}\text { la economía } \\
\text { problemas } \\
\text { económicos }\end{array}$ \\
\hline 2011 & SEGURIDAD & SEGURIDAD & salud & SEGURIDAD & SEGURIDAD & EMPLEO & $\begin{array}{l}\text { la economía/ } \\
\text { problemas } \\
\text { económicos }\end{array}$ \\
\hline 2013 & SEGURIDAD & SEGURIDAD & salud & SEGURIDAD & SEGURIDAD & SEGURIDAD & SEGURIDAD \\
\hline 2015 & SEGURIDAD & SEGURIDAD & corrupción & SEGURIDAD & $\begin{array}{l}\text { desabastecimiento } \\
\text { falta de alimentos } \\
\text { acaparamiento }\end{array}$ & EMPLEO & SEGURIDAD \\
\hline
\end{tabular}

Fuente: Latinobarómetro.

${ }^{12}$ En el año 1995 el estudio no se llevó a cabo en Ecuador y Bolivia, por ello se coloca la referencia S/D (Sin dato). 
Lo primero a destacar es que la media latinoamericana es la que más se parece a la tendencia histórica de la Argentina y viceversa. Ningún otro país muestra una evolución de temas tan similar al promedio continental, donde la única diferencia observada es que en Argentina el agrupamiento vinculado a inseguridad emerge como principal en el 2007 mientras que para el conjunto latinoamericano lo hace al año siguiente (2008).

Si tomamos el grupo de países "posneoliberal-desarrollista" observamos que, efectivamente, Argentina y Uruguay muestran una evolución de agendas muy similar: en el país oriental la principalidad de la cuestión de la inseguridad se hace presente algunos años después (2009), pero a partir de allí se instala con fuerza. En tanto que Brasil resulta coincidente con los problemas señalados en nuestro país hasta el año 2009, pero, a partir del 2010, cuenta con una agenda bien diferente al resto del continente, emergiendo el tema de la salud entre 2010/2013 y el de corrupción en 2015. Esto último resulta comprensible si tomamos en consideración las denuncias por corrupción que afrontó el gobierno brasileño en ese año y que derivaron recientemente en un proceso de juicio político contra la presidenta Dilma Rousseff.

Por su parte, al considerar el conjunto de "países en transición" encontramos situaciones divergentes. Si bien Venezuela hasta el año 2000 mostraba un predominio en su agenda de "otros temas" tales como inflación (1995) y educación (1996-2000); a partir de 2001 pareciera tener un comportamiento más parecido al de Argentina y Uruguay que a los otros países de su grupo. La excepción se produce en la última medición (2015), cuando, a raíz de la fuerte crisis vinculada al desabastecimiento de alimentos, este tema particular emerge con fuerza en dicho país.

Entre tanto Ecuador y Bolivia parecerían mostrar una evolución similar, en la que la cuestión del empleo se impone en el centro de la agenda por mayor cantidad de años y los temas vinculados a la inseguridad parecerían emerger de forma más tardía que en el resto de los países analizados. Es de destacar también la presencia en estos dos paí- 
ses de la categoria "Situación política/Problemas de la política", algo poco señalado en general por la opinión pública como principal problema de un país y cuya mención mayoritaria habitualmente denota situaciones de inestabilidad y/o de fuertes confrontaciones en este terreno. En Ecuador esto ocurre en 2005, cuando se produce la caída del expresidente Lucio Gutiérrez; por su parte, en Bolivia, la mencionada categoría obtiene mayor cantidad de menciones en 2008, año de la fuerte crisis interna por los levantamientos contra el presidente Evo Morales en la región de la Media Luna.

En definitiva, podemos concluir que la construcción analítica de los agrupamientos de países en función del tipo de gobierno y del proceso político-social por el que atraviesan permite identificar una correlación parcial con los temas de agenda señalados por sus respectivas poblaciones en el estudio Latinobarómetro.

Este entrecruzamiento de análisis y datos enfocados desde diferentes dimensiones de la realidad social nos permite consolidar algunas hipótesis y explicaciones sobre la similitud de determinados procesos y seguir reflexionando sobre otros que parecerian conllevar otro nivel de complejidad.

\section{¿Y el resto del mundo?}

Nos hemos restringido hasta aquí a comparar datos del Latinobarómetro obtenidos en Argentina con los de otros países y el conjunto de América Latina, confirmando con ellos que el fenómeno de emergencia del tema seguridad/inseguridad es parte de una tendencia continental. Pero surge el interrogante de si no se trata en verdad de una cuestión global, que excede incluso los marcos de nuestro continente. Para intentar dar respuesta a esto no podemos apoyarnos en los resultados de este mismo estudio, puesto que este se restringe a América Latina. Como forma de aproximación al tema expondremos a continuación los resultados del índice Law and Order de Gallup World Poll. ${ }^{13}$

\footnotetext{
${ }^{13}$ http://www.gallup.com/poll/175082/latin-america-scores-lowest-security.aspx
} 
El mencionado indicador se propone medir la sensación de seguridad de los ciudadanos a partir de tres variables: confianza en la policía local, sentimiento de seguridad personal y autorreporte de robos. Los resultados se obtienen del estudio anual de opinión pública (World Poll) que realiza la consultora Gallup, el que alcanza a más de 160 países, constituyéndose así en el estudio continuo de opinión publica de mayor alcance a nivel global del que se tenga conocimiento hasta la fecha. ${ }^{14}$

En el Cuadro 2 podemos observar los resultados del índice Law and Order comparados a nivel continental, donde se destaca que América Latina y el Caribe obtiene el puntaje más bajo tanto en 2009 como en 2013. En otros términos, es en nuestro continente donde los entrevistados manifiestan mayor sensación y experiencias de inseguridad así como de desconfianza en las instituciones encargadas de garantizarla y/o dar respuesta a ella.

Cuadro 2: Índice Law and Order de Gallup World Poll

\begin{tabular}{|l|c|c|}
\hline & 2009 & 2013 \\
\hline Southeast Asia & 82 & 80 \\
\hline East Asia & 79 & 80 \\
\hline U.S. and Canada & 80 & 79 \\
\hline Europe & 76 & 77 \\
\hline South Asia & 76 & 70 \\
\hline Middle East and North Africa & 70 & 65 \\
\hline Former Soviet Union & 56 & 62 \\
\hline Sub-Saharan Africa & 60 & 59 \\
\hline Latin America and the Caribbean & 54 & 56 \\
\hline
\end{tabular}

Fuente: Gallup.

\footnotetext{
${ }^{14}$ En la Argentina se realizan anualmente 1000 encuestas con una muestra nacional, representando a todos los estratos de población y con un método de selección aleatorio del entrevistado al interior del hogar (sorteo mediante tabla kish). En la Argentina este estudio es realizado desde 2005 por MBC MORI.
} 
No pretendemos con esto postular afirmaciones concluyentes, pero debemos mencionar que estos datos al menos apoyan una intuición expuesta desde un comienzo que nos indicaba la presencia de un fenómeno que excedía los marcos de nuestro país, pero situándose en un plano continental.

Junto con esta afirmación quedan entonces planteados una serie de interrogantes y desafíos a futuro. Entre otros podemos mencionar: a) ¿Cuáles son las causas de la emergencia de este tema en América Latina?; b) ¿Por qué su instalación se produce de forma más pronunciada en nuestra región que en el resto del mundo?; c) iSe trata de un fenómeno pasajero o de una tendencia de largo alcance que puede derivar a futuro en reconfiguraciones societales profundas?

Estos son solo algunos de los interrogantes que el presente análisis nos deja planteados y que pensamos pueden ayudarnos a comprender mejor las causas, el alcance y, sobre todo, las consecuencias de la emergencia y consolidación del binomio seguridad/inseguridad como preocupación de primer orden público en nuestro país.

\section{Referencias}

Elorza, Enrique (2015) Economía Politica en la Transición. Ir hacia una mesa servida para todos en Nuestramérica. Buenos Aires: Fundación de Investigaciones Sociales y Politicas (FISyP).

Gambina, Julio C. (2013) Crisis del capital 2007/2012. La crisis capitalista contemporánea y el debate sobre las alternativas. Buenos Aires: Fundación de Investigación Sociales y Politicas (FISyP).

Kessler, Gabriel (2011) "La extensión del sentimiento de inseguridad en América Latina: relatos, acciones y politicas en el caso argentino". Revista de sociología e política.

Kessler, Gabriel (2008) "Sentimiento de inseguridad y miedo al crimen en Argentina", en V Jornadas de investigación en Antropología social. Buenos Aires: Facultad de Filosofía y Letras UBA. 
Sozzo, Máximo "¿Contando el delito? (2003). Análisis crítico y comparativo de las encuestas de victimización en Argentina". Universidad Nacional del Centro. En https://www.oas.org/dsp/ documents/victimization_surveys/argentina/Argentina\%20\%20Analisis\%20critico\%20y\%20comparativo\%20de\%20 las\%20Encuestas\%20de\%20Victimizacion.pdf

Resultados del estudio sistemático de Opinión Pública Latinobarómetro. http://www.latinobarometro.org/lat.jsp

Resultados del Latin America Scores Lowest on Security, en: http://www.gallup.com/poll/175082/latin-america-scoreslowest-security.aspx

Resultados del estudio LAPOP en: http://datasets.americasbarometer. org/database-login/index.html 